UDC: 616.34-002.4-072

DOI: 10.26697/ijes.2019.4.48

\section{The Effectiveness of Transchiatal Esophagoectomy}

Associate Professor Shevchenko A. N. ${ }^{\mathbf{1}}$, Associate Professor Bityak S. Y. ${ }^{\mathbf{1}}$, Polikov H. O. ${ }^{1}$

${ }^{1}$ Kharkiv National Medical University, Ukraine

\begin{abstract}
Background:

Today the number of patients with cancer continues to increase. Esophageal cancer one of the most difficult disease in terms of diagnosis and treatment, in which surgical treatment remains the fundamental therapy. There are many methods, which divided into 2 large categories: transthoracic and transchiatal. Discussions about effectiveness of the techniques are going on. It should be noted that transchiatal access reduces mortality from the effects of thoracotomy.

Among oncological diseases, cancer of the esophagus takes 8th place. It is important to note the high mortality rate from cancer of the esophagus -350000 patients every year. The priority in modern surgery for patients with esophageal cancer with functional disorders of the cardiovascular and respiratory systems is the use of transhiatal access. Extensive metaanalytical studies have been carried out in 1996 Ringani et al. and in 2002 Hulscher et al.
\end{abstract}

\section{Methods:}

For the period 2015-2018 at "V. T. Zaitsev institute of general emergency surgery" 24 patients with adenocarcinoma $(75.0 \%)$ and squamous cell carcinoma (25.0\%) underwent resection of the esophagus with simultaneous plastic surgery of the stomach stem. A set of examinations was carried out: endoscopic and radiological examinations, clinical analysis of blood and urine, a coagulogram, a biochemical blood test, a preoperative examination of therapist and anesthesiologist was carried out, and the patient's preoperative status was determined according to the ASA scale. The gender ratio is 15 men and 9 women. The average age is $36 \pm 5$ years. The average body mass index is 20.3. Among patients there were no people with bad habits. All patients were divided into 2 main groups according to the method of surgical intervention: group I - transhiatal (50.0\%); II transthoracic $(50.0 \%)$.

\section{Results:}

The advantages of transchiatal access are the absence of thoracotomy, which reduces the duration of postoperative pain and postoperative complications. The duration of the surgical intervention was: with transhiatic access (I) an average of $180 \pm 30.0$ minutes; group II - $360 \pm 25.0$ minutes. Intraoperative blood loss was in the I group - $1000 \pm 25.0 \mathrm{ml}$, II $-2000 \pm 44.1$ $\mathrm{ml}$.

All patients of group I switched to independent breathing and disconnected from the mechanical ventilation on the 1 st day after surgery. Among patients in the control group, this indicator in $35.0 \%$ of patients increased to 2 days. It is important to note that in patients of group II, hemodynamic instability was noted - an increase in blood pressure with poorly amenable to correction. In group I, according to the indicators of daily ECG monitoring, there is a lesser degree of ischemic changes in the myocardium: an increase in the control group by $35.3 \%$ of episodes of ST segment depression, and the degree of ST depression by $16.7 \%$. In the early postoperative period, mortality in the main group was 2 patients (the cause of death was acute respiratory and heart failure), in the control group - 4 (1 patient had interstitial pulmonary edema, 2 - acute heart failure, 1 - acute respiratory failure). The confidence is $\mathrm{p}<0.05$.

The main cause of death in the early postoperative period among patients with esophageal cancer was a sharp disadaptation of the cardiovascular and respiratory systems.

\section{Conclusions:}

The use of transhiatric access reduces the length of stay of patients in intensive care. It allows you to expand the indications in patients with functional disorders of the cardiovascular and respiratory systems, significantly reduce the number of deaths in the postoperative period by $8.3 \%$ compared with the control group.

\section{Information about the authors: \\ Shevchenko Oleksandr Mykolajovich - Doctor of Philosophy in Medicine, Associate Professor of Department of Surgery No. 1, Kharkiv National Medical University, Kharkiv, Ukraine. \\ Research interests: education and training, methodology and theory of surgery; https://orcid.org/0000-0002-1176-1687.}

Bityak Sergey Yuryevich - Doctor of Philosophy in Medicine, Associate Professor of Department of Surgery No. 1, Kharkiv National Medical University, Kharkiv, Ukraine.

Research interests: education, methodology and theory of surgery; https://orcid.org/0000-0002-6012-2048.

Polikov Heorhii Olegovych - Kharkiv National Medical University, Kharkiv, Ukraine.

Research interests: education, social pedagogy, theory of surgery, culture of health; https://orcid.org/00000001-9232-4667.

Corresponding Author:

Shevchenko Oleksandr Mykolajovich

Corresponding Author's Email:

knmu.surgery@gmail.com 